\title{
Knowledge acquisition for reactive scheduling
}

\author{
B.J. Garner and G.J. Ridley \\ School of Computing and Mathematics, \\ Deaking University, Geelong, Victoria 3217, Australia \\ Tel: +61 52271383 Fax: -+61 52272028 e-mail (brian.riddles)@deakin.edu.au
}

\begin{abstract}
In this paper we report on the development and use of a tool for the acquisition of heuristics used by operators of a glass coater in reactive scheduling. These results complement previous studies of neural network process models in scheduling production for the glass coating operation (AIRCO) at Pilkington Australia. Integration of our knowledge rich control strategies in the construction of an Expert Adviser for scheduling is now in progress.
\end{abstract}

Keyword Codes:

Keywords: Knowledge Acquisition, Reactive Scheduling, Heuristics, AIRCO.

\section{INTRODUCTION}

In our studies of knowledge-based reactive scheduling for the glass-coating operation (AIRCO process, 1988), we identified the value of neural network process models for scheduling production (Garner \& Ridley, 1994). This phase of our research also identified knowledge rich control strategies for reactive scheduling, which require an understanding of the heuristics employed by the glass coating operators in response to changes in management priorities and to constraints (bottlenecks) in the production process.

The glass-coating operation is conducted at Pilkington's Dandenong plant and uses an Ion-implantation method to tint architectural glass. The ionimplantation is achieved using a vacuum technology that is proprietary to the AIRCO company. The coating system is a continuous process in which the glass enters a series of high vacuum coating chambers.

The coating chambers contain specially designed cathodes and process gases. The coating process is controlled by varying the power applied to each of the cathodes and by adjusting the flow rate of the process gases. 
The colour of the coated glass is measured online after the coating has been completed. This is presently done by a spectrophotometer that is manufactured by an American company called Macbeth. The spectrophotometer (Macbeth) is connected to a host computer (an IBM PS/2) which initialises, displays, and stores the measurements from the spectrum anylyser. When coated glass travels over the Macbeth, 3 measurements are taken (characterised by 3 bright flashes) to determine the average spectral properties of the glass.

In this paper, we now report on the problem of maintaining control in an environment where the predetermined schedule of jobs is frequently interrupted. Possible reasons for such interruptions include:

- urgent orders of replacement glass to replace panes broken or damaged in a large order; and

- a suspension to coating to conduct minor maintenance, such as a burn-in, or a reassignment of job priorities by management.

The heuristics used by operators for reactive scheduling are not immediately obvious, and while substantial progress in the data engineering phase of the project (Garner \& Ridley, 1993) resulted in new insights into the knowledge acquisition requirements, the discovery of appropriate heuristics had to wait on the establishment of a cumulative database of coating histories and process control information. The massive database collected over time during AIRCO coating operations now supports determination of:

- control settings used in previous coatings;

- $\quad$ settings that resulted in the best coatings;

- cathode usage between coatings or since a particular coating;

- current coating performance in comparison to previous results.

The knowledge acquisition tool relies on access to the database for retrieval of selected coating histories and provides a novel interface to the operator in understanding the basis of decisions made in a particular shift and the consequences (simulation mode) of those decisions.

Recent studies (comparative analysis) by the authors of neural network process models for predictive control of the progress of the coating of glass identified the Generalised Regression Neural Network (GRNN), developed by Donald Specht, as a suitable control aid. The GRNN is a memory-based neural network, that has a one-pass learning algorithm. The attraction of the GRNN developed for glass coating lies in the speed at which a continuous model can be constructed and the smooth transition between observed values.

Back propagation, by contrast, (Bhat et al, 1990) while capable of handling the non-linearities in spectral data, has a substantial disadvantage in the time taken to retrain such networks, and there is no guarantee of convergence. 


\section{KNOWLEDGE ACQUISITION PROCESS}

For any particular time frame (i.e. shift), the operator is subject to certain priorities (goals), constraints on glass availability (throughput) from other processes, and physical constraints within the AIRCO process. Our understanding of this constrained set of goals is translated into a set of propositions, from which control rules are derived and, which are used for extracting coatings for scheduling. These coatings are extracted from the coating history database and reflect, as far as possible, the realistic environment that the operator would be facing at that instance. The tool for displaying information to the operator on these jobs is a graphic tool and facilitates scheduling by the operator to produce optimum schedules. The knowledge acquisition process is now required to infer the rules used by the operator from the schedules produced. Naturally, due to the uncertainty in the constrained goals from which the propositions were derived, it is necessary to iterate until a stable set of rules has been produced. This iterative process is guided by metaknowledge in the form of a set of axioms, which are discussed elsewhere. The overall schematic for the knowledge acquisition process is shown below in Figure 1:

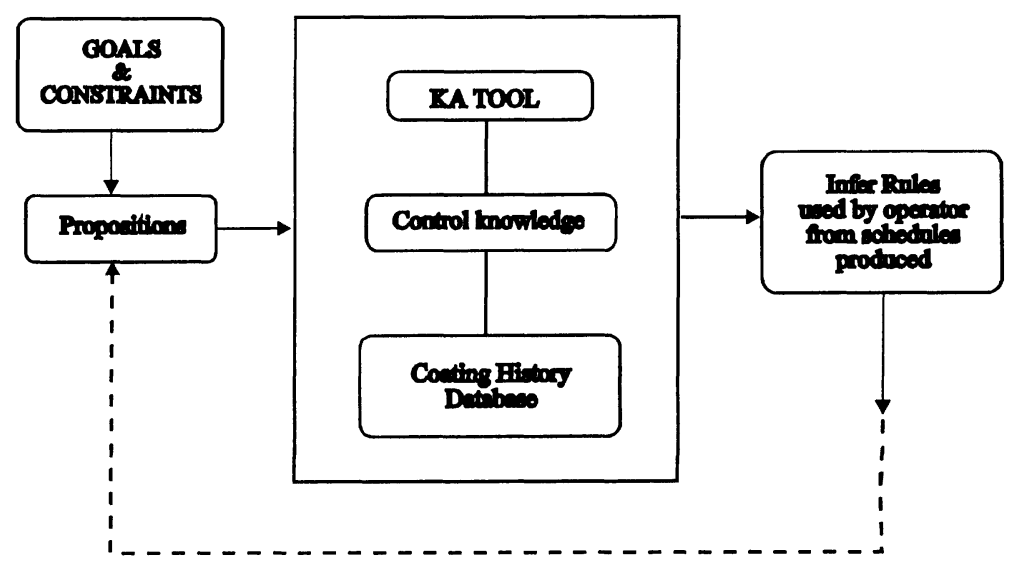

Figure 1. Knowledge Acquisition (KA) Process

The control considerations involve such factors as;

- time since the previous maintenance call

- continuous wear and tear on cathodes

- large sheets of glass cut the gas supply, so it would be necessary to reduce the power supply.

- TS coatings are relatively easy to reproduce because they use 6 cathodes, which gives more flexibility (i.e. one cathode may be worn, so increase power on another!) 
- Coatings that use oxygen in the coating chamber are generally harder to control.

- The degree of difficulty ascribed by operators to coatings reflect the flexibility and reproduceability of the coatings.

Note: The Macbeth instrument for measuring spectral data will give false values if the glass size is small ( $3 \mathrm{rd}$ flash misses). Operator sometimes temporarily slows linespeed over Macbeth, so that 3rd flash measures glass. Linespeed must then be returned to normal to avoid build up of glass in exit chamber. The operator my be concerned that on large sheets the Macbeth is only measuring one end of the sheet and not taking measurements along whole length of sheet.

- Operators pay equal attention to $\mathrm{L}$, $\mathrm{a}$ and $\mathrm{b}$ values. If $\mathrm{L}$ can be controlled, then a \& b would normally follow.

Rules derived from these control considerations are summarised below in point form:

- On simple coatings increasing Power increases L, a\&b.

- On simple coatings decreasing Power reduces $\mathrm{L}$, a\&b.

- Don't adjust the AIRCO machine unless a $0.2 \%$ change in $\mathrm{L}$, $\mathrm{a}$ or $\mathrm{b}$ is necessary.

- During a simple coating, little change is necessary to the machine following the initial setup. This means that lineups are critical in determining which chamber coating is behaving erratically.

- Interstage transmission monitors enable monitoring of progress in each chamber. Monitors are useful in determining for which chambers adjustments are necessary

- An increase in power is always required, if no maintenance has been conducted, to reproduce a particular coating.

- Behaviour of cathodes is independent of controller position.

- Operators use flow rate control rather than pressure control.

- Changing bottles (some gas) has no effect on coating.

\section{PURPOSE AND BENEFITS OF THE KNOWLEDGE ACQUISITION TOOL}

The purpose of the knowledge acquisition tool is to present to the operator a list of jobs (i.e. customer orders) to be processed in the time frame in question, usually a complete shift, and to assist through visual display, production of the actual schedule. The tool appeals to the operators due to the touch and feel 
quality of the user interface. The AIRCO operator may be presented with up to 15 customer orders generated from the database, which may of course, be randomly selected, for rearrangement of the desired schedule. Where customer orders are selected randomly, the variables of interest are typically:

- Customer

- Coating type

- Glass size

- Glass thickness

- Number of sheets (random number between 1 and 99)

Note that the customer orders generated are not simply selections from the database, although they could be, but would typically be new types of jobs based on the populations of the various parameters of interest. This unique feature of the tool enables the operator to study new situations, which may not have been encountered before, and to advise on how the new parameters would influence the possible schedule.

The attraction of the knowledge acquisition tool to the operator lies very much in the graphical interface, in which the customer orders appear as a list of pending jobs. As each job is selected, it is removed from the list and appended to the list of scheduled jobs. The final list is, of course, a schedule of jobs in the desired order for coating. Comments are encouraged by the operator in clarification of the final schedule, and such explanations in clarification of the selection, are valuable in the rule inference phase of the knowledge acquisition. The scheduled orders and comments are saved to file for future reference.

\section{INFERRING RULES FROM TASK SCHEDULES}

The schedules and associated comments generated by the operator from use of the tools are examined offline, and in consultation with the operator, rules are extracted for use in the expert adviser. In general terms, rules may be generic, may be highly specific, depending upon the coating requirements, or may be restricted in terms of the jobs to be included in a particular batch. For example, the post-processing of tinted glass is a major factor in the scheduling of customer orders, and is usually determined by physical size, fitness and quantity of the glass. The grouping of similar types of glass coatings is a lower priority than size considerations, even though this increases the number of set-point changes.

To illustrate the process of heuristic discovery, reference is made to the following list of jobs that were generated by the tool and scheduled by the AIRCO operator. The jobs appear in the desired order of coating. These results, shown in Figure 2, permitted the discovery that the SS8 CLR coating is a special case. It violated a previous heuristic that stated that all $3 \mathrm{~mm}$ and $6 \mathrm{~mm}$ coatings should be grouped together! From further investigation with the operator, it was shown that the SS8 coating should always be scheduled after a burn-in (and a 
burn-in is always conducted before the commencement of the shift to clean the machine). Thus, scheduling an SS8 after other coatings was seen to increase the risk of contamination of the glass surface! In practice, the $6 \mathrm{~mm}$ SS8 CLR was chosen first, so that all $3 \mathrm{~mm}$ coatings could be grouped together, but also because of a second heuristic; namely, the operator prefers to do large orders first!!

\begin{tabular}{llllll}
\hline \multicolumn{2}{c}{ Customer } & Thick & Coating & Size & No Ordered \\
\hline PDFAWK & MELB & $6 \mathrm{MM}$ & SS8 CLR & $3657 * 2134$ & 70 \\
\hline PDOAKL & MELB & $3 \mathrm{MM}$ & SS8 CLR & $3048 * 2134$ & 40 \\
\hline PDSA & MELB & $3 \mathrm{MM}$ & SS8 CLR & $3048 * 2134$ & 44 \\
\hline PDHUME & CANB & $3 \mathrm{MM}$ & TS30 CLR & $3657 * 2134$ & 55 \\
\hline PDTAMW & SYDN & $3 \mathrm{MM}$ & TS40 CLR & $3657 * 2134$ & 97 \\
\hline PDOAKL & MELB & $3 \mathrm{MM}$ & SL10 & $3657 * 2134$ & 81 \\
\hline PDOAKL & MELB & $6 \mathrm{MM}$ & SS14 CLR & $3048 * 2134$ & 70 \\
\hline PDSA & ADEL & $6 \mathrm{MM}$ & TS21 CLR & $3048 * 2134$ & 16 \\
\hline PDSA & ADEL & $6 \mathrm{MM}$ & TS21 CLR & $3048 * 2134$ & 44 \\
\hline PDFAWK & MELB & $6 \mathrm{MM}$ & TS30 CLR & $3048 * 2134$ & 52 \\
\hline PDHUME & CANB & $6 \mathrm{MM}$ & TS30 CLR & $3048 * 2134$ & 11 \\
\hline PDOAKL & MELB & $6 \mathrm{MM}$ & SS22 CLR & $3048 * 2134$ & 13 \\
\hline PDSA & ADEL & $6 \mathrm{MM}$ & SL22 & $3048 * 2134$ & 2 \\
\hline PDHUME & CANB & $6 \mathrm{MM}$ & SL22 & $3048 * 2134$ & 11 \\
\hline
\end{tabular}

Comments: The SS8 $6 \mathrm{~mm}$ order is processed first as it has the largest number of sheets. The SL order is processed last as it subsequently requires laminating.

Figure 2.

A further heuristic discovered from the schedule shown in Figure 2 was that the SL family of coatings should always be done last. This follows from the need to send the product to another part of the factory. The despatch is easier to manage if they are last on the frame (a frame is the method of storing glass once it has been coated and has a last-in first-out logic).

\section{VERIFICATION OF SCHEDULING RULES}

Given the uncertainties in goals and constraints encountered in reactive scheduling, the verification of scheduling rules produced from the schedules 
provided by the operators was regarded as crucial. Three phases in the verification process were implemented:

\section{A. Selection from past customer orders}

The customer orders in this phase were selected directly from the database and based on the operators' schedules, the repeatability of the previous schedules was verified directly.

\section{B. Random generation of jobs}

In this phase each field was randomly generated within the range permitted and a job constructed accordingly. This approach enabled us to tease out how the operator schedules jobs which they have not previously encountered or jobs which they have not encountered in the particular combinations now presented. The operator is thus forced to confront new situations and to provide such rules as they can. These rules can then be compared with other rules to determine whether the normal consistencies or contradictions exist?

\section{User defined list}

The third phase of verification permits the user to define a list and enables the knowledge engineer to add extra constraints to a previous set of jobs: For example, the last 8 cases of $6 \mathrm{~mm}$ glass in Figure 3-5 are all the same size. How should the operator schedule these jobs if the size of some of the 3048 is changed 3657? The importance of sheet size in the scheduling heuristics was discovered in the third phase of verification. For example, in the first interview a number of jobs were generated randomly and the schedule logged. In the second interview, the same set of jobs were presented to the operator, but the number of sheets (i.e. the number ordered) was changed. The same schedule was received. In the third interview the number of jobs was the same as in the other two interviews other than a change in sheet size. In this case, a different schedule was obtained!

The operators of the AIRCO process have expressed strong support for the method of knowledge acquisition used in this study. The evaluation process is continuing in conjunction with the operators. Requests from them for modifications to accept a list of pending jobs, as generated by the customerservice department, have now been made. The tool now permits the operator to sort jobs for commencement of coating and in this way, we expect to proceed to an automated presentation step, in which the tool is able to provide provisional schedules by sorting for the operators using rules previously extracted, with minor subsequent changes to accommodate specific (new) external constraints. This verification of the acquired rules through the automated presentation tool is, we believe, a final but essential step in construction of the Expert Adviser. 


\begin{tabular}{|c|c|c|c|c|c|c|}
\hline "PDEAWK & MELB & $6 \mathrm{MM}$ & SS8 & CLR & $3657 * 2134$ & $70 "$ \\
\hline "PDOAKL & MELB & $3 \mathrm{MM}$ & SS 8 & CLR & $3048 * 2134$ & $40 "$ \\
\hline "PDSA & ADEL & $3 \mathrm{MM}$ & SS 8 & CLR & $3048 * 2134$ & $44 "$ \\
\hline "PDHUME & CANB & $3 \mathrm{MM}$ & TS30 & CLR & $3657 * 2134$ & $55^{\prime \prime}$ \\
\hline "PDTAMW & SYDN & $3 \mathrm{MM}$ & TS40 & CLR & $3657 * 2134$ & $97 "$ \\
\hline "PDOAKL & MELB & $3 \mathrm{MM}$ & SL10 & & $3657 * 2134$ & $81 "$ \\
\hline "PDOAKL & MELB & $6 \mathrm{MM}$ & SS14 & CLR & $3048 * 2134$ & $70^{\prime \prime}$ \\
\hline "PDSA & ADEL & $6 \mathrm{MM}$ & TS21 & CLR & $3048 * 2134$ & $16 "$ \\
\hline "PDSA & ADEL & $6 \mathrm{MM}$ & TS21 & CLR & $3048 * 2134$ & $44^{\prime \prime}$ \\
\hline "PDEAWK & MELB & $6 \mathrm{MM}$ & $\mathrm{TS} 30$ & CLR & $3048 * 2134$ & $52 "$ \\
\hline "PDHUME & CANB & $6 \mathrm{MM}$ & TS30 & CLR & $3048 * 2134$ & $11 "$ \\
\hline "PDOAKL & MELB & $6 \mathrm{MM}$ & SS22 & CLR & $3048 * 2134$ & $13 "$ \\
\hline "PDSA & ADEL & $6 \mathrm{MM}$ & SL22 & & $3048 * 2134$ & $2 "$ \\
\hline "PDHUME & CANB & $6 \mathrm{MM}$ & SL22 & & $3048 * 2134$ & $11 "$ \\
\hline
\end{tabular}

Figure 3.

\begin{tabular}{|c|c|c|c|c|c|c|}
\hline "PDFAWK & MELB & $6 \mathrm{MM}$ & SS 8 & CLR & $3657 * 2134$ & $30^{\prime \prime}$ \\
\hline "PDOAKL & MELB & 3MM & SS 8 & CLR & $3048 * 2134$ & $40^{\prime \prime}$ \\
\hline "PDSA & ADEL & $3 \mathrm{MM}$ & SS 8 & CLR & $3048 * 2134$ & $44 "$ \\
\hline "PDHUME & CANB & $3 \mathrm{MM}$ & TS30 & CLR & $3657 * 2134$ & $55^{\prime \prime}$ \\
\hline "PDTAMW & SYDN & 3MM & TS 40 & CLR & $3657 * 2134$ & $97 "$ \\
\hline "PDOAKL & MELB & 3MM & SL10 & & $3657 * 2134$ & $81 "$ \\
\hline "PDOAKL & MELB & $6 \mathrm{MM}$ & SS14 & CLR & $3048 * 2134$ & $70^{\prime \prime}$ \\
\hline "PDSA & ADEL & $6 \mathrm{MM}$ & TS21 & CLR & $3048 * 2134$ & $20 "$ \\
\hline "PDSA & ADEL & $6 \mathrm{MM}$ & TS21 & CLR & $3657 * 2134$ & $40^{\prime \prime}$ \\
\hline "PDFAWK & MELB & $6 \mathrm{MM}$ & TS30 & CLR & $3657 * 2134$ & $52 "$ \\
\hline "PDHUME & CANB & $6 \mathrm{MM}$ & TS30 & CLR & $3048 * 2134$ & $11 "$ \\
\hline "PDOAKL & MELB & $6 \mathrm{MM}$ & SS22 & CLR & $3048 * 2134$ & $13^{\prime \prime}$ \\
\hline "PDSA & ADEL & $6 \mathrm{MM}$ & SL22 & & $3657 * 2134$ & $20 "$ \\
\hline "PDHUME & CANB & $6 \mathrm{MM}$ & SL22 & & $3048 * 2134$ & $11 "$ \\
\hline
\end{tabular}

Figure 4.

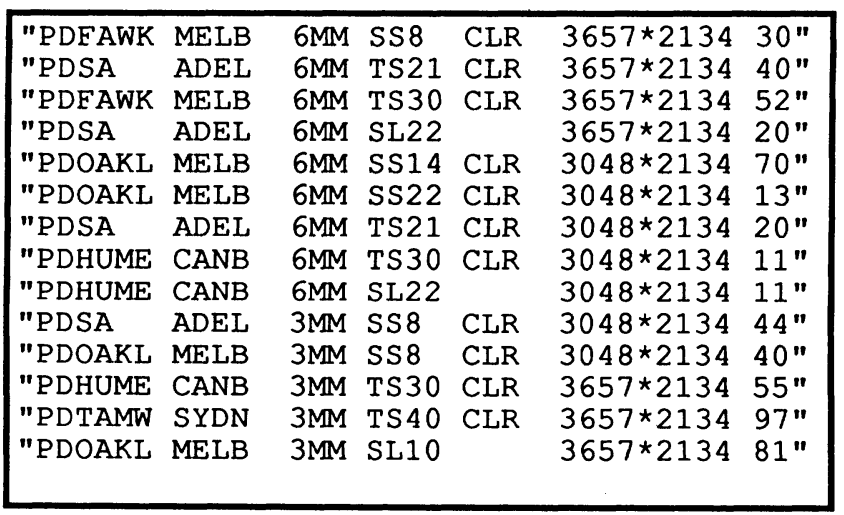

Figure 5

Effect of Glass Size on Schedule 


\section{FUTURE RESEARCH}

The research is continuing with four key areas of investigation.

i. The validation of heuristics obtained from the knowledge acquisition described above is on-going and will naturally address such issues as rule normalisation and metaknowledge requirements.

ii. Extension of our preliminary studies of neural network process models for scheduling production of the glass coating operation has increased our knowledge of process interactions, and it is now possible to construct a model of the coating process for individual coatings. This work now needs to be finalised for inclusion in the Expert Adviser.

iii. The links between the database, heuristics, and the neural network process model require further work. The objective is to index the database such that training examples may be rapidly recalled for the ANN model in response to particular heuristics.

iv. The final contribution lies in the area of control knowledge for the Expert Adviser, in which all components are integrated in providing an advisory system to the operators. The intention is to further develop the knowledge acquisition tool described in this paper for the capture of control knowledge governing the proposed interactions with the operators.

\section{ACKNOWLEDGMENTS}

This research is supported by Pilkington Australia and the Australian Research Council APRA Scholarship (Industry) Grant number 2G90/342

\section{REFERENCES}

AIRCO, (1989); Process Theory and Practice, GR-20, AIRCO operations manual, AIRCO Coating Technology, U.S.A., (1989), Chapter 4.

B.J. Garner and G. J. Ridley and P. J. Lower, Data Engineering for Neural Net Analysis of Glass Furnace Characteristics. Proceedings of the ANNES Conference, New Zealand, (1993).

B.J. Garner and G. J. Ridley, Application of Neural Network Process Models in Reactive Scheduling. Proceedings of the IFIP TC5/WG5.7 Internatinal Workshop on Knowledge-Based Reactive Scheduling; Athens, Greece, (1994).

N. V. Bhat, P.A. Minderman, Jt, T. McAvoy, and N. S. Wang, Modelling Chemical Process Systems via Neural Computation, IEEE Control Systems Magazine, April, (1990),24.

D. F. Specht, A General Regression Neural Network, IEEE Transactions on Neural Network, November, (1991), 568. 